\title{
SOME REMARKS ON THE POMPEIU PROBLEM FOR GROUPS
}

\author{
DAVID SCOTT AND ALLADI SITARAM \\ (Communicated by David G. Ebin)
}

\begin{abstract}
A Borel set $E$ in a topological group $G$ is said to be a $P$-set for the space of integrable functions on $G$ if the zero function is the only integrable function whose integral over all left and right translates of $E$ by elements of $G$ is zero. For a "sufficiently nice" group $G$ and a Borel set $E$ of finite Haar measure a certain condition on the Fourier transform of a function related to $E$ is shown to be a sufficient condition for $E$ to be a $P$-set. This condition is then applied to several classes of groups including certain compact groups, certain semisimple Lie groups, the Heisenberg groups and the Euclidean motion group of the plane.
\end{abstract}

1. Introduction. Let $(X, \beta, \mu)$ be a measure space on which a group $H$ acts as a group of measure preserving transformations. The following kind of question has come to be known in the literature as the "Pompeiu problem" (see [Z]): Given a class $C$ of measurable functions and a measurable set $E \subset X$ (of, say, positive finite measure), when can you "recover" a given $f \in C$ from the "data" $\int_{g E} f d \mu$, $g \in H$ ? A simpler version of this question is: If $f \in C$ and $\int_{g E} f d \mu=0 \forall g \in H$, is $f=0$ ? This problem has been studied in detail when (a) $X=\mathbf{R}^{n}$ and $H$ is either the group of translations or the group of rigid motions (see for example [Ba-Si, Be I, Br-S-T, Sa and Si I], and when (b) $X$ is a symmetric space and $H$ a transitive group of isometries of $X$ (see for example [Be-Sh, Be-Z, R I and Si II]). Another case which is worth studying is: $X$ a locally compact unimodular group, $\mu$ the Haar measure on $X$, and $H$ the group of two-sided translations on $X$. Some progress on this problem has been made by I. K. Rana (see [R II]), who deals mainly with the locally compact abelian group situation. In [Si II] the case when $X$ is a noncompact semisimple Lie group is taken up very briefly. There it is shown that, unlike the case of $\mathbf{R}^{n}$ or symmetric spaces of the noncompact type, for $G=S L(2, \mathbf{R})$, there exist relatively compact sets $E$ with positive Haar measure and nontrivial $f \in L^{1}(G)$ with the property that $\int_{g E} f d \mu=\int_{E g} f d \mu=0 \forall g \in G$. Actually, if one considers two sided translations, a more natural question to ask is the following: Do there exist $E$ and $f \neq 0$ as above such that $\int_{g_{1} E g_{2}} f d \mu=0 \forall g_{1}, g_{2} \in G$ ? It can be proved that the example given in $[\mathbf{S i} \mathbf{I I}]$ actually satisfies this slightly stronger property.

The purpose of this paper is to consider the case when $X$ is a unimodular group $G, H$ is the group of two-sided translations of $G$ on itself, and $C=L^{1}(G, \mu)$ with $\mu$ the Haar measure on $G$. After proving (in $\S 3$ ) sufficient conditions for a set $E$ to be a $P$-set (see $\S 2$ for the definition), we examine some concrete situations where the

Received by the editors December 1, 1987.

1980 Mathematics Subject Classification (1985 Revision). Primary 22E30, 22D99; Secondary $43 \mathrm{~A} 80$. 
conditions can be verified (semisimple Lie groups, Heisenberg groups, the Euclidean motion group on the plane and compact groups).

2. Notation and preliminaries. Throughout this paper $G$ will denote a "sufficiently nice" locally compact unimodular group and $\hat{G}$ its unitary dual, that is, $\hat{G}$ is a maximal set of pairwise inequivalent irreducible unitary representations of $G$. We assume that, for the class of groups we are dealing with, I. Segal's abstract Plancherel theorem is valid (see [Wa]). Fix a Haar measure $\mu$ on $G$ and let $m$ be the corresponding Plancherel measure on $\hat{G}$. $E$ will denote a Borel subset of $G$ with $0<\mu(E)<\infty$ and $\tilde{E}=\left\{g \in G: g^{-1} \in E\right\}$. Let $1_{E}$ and $1_{\tilde{E}}$ denote the indicator functions of $E$ and $\tilde{E}$ respectively. Note that $1_{E}$ and $1_{\tilde{E}}$ are bounded measurable functions and are in every $L^{p}(G, \mu)$ for $1 \leq p \leq \infty$. For $f \in L^{1}(G)$ and $\pi \in \hat{G}, \hat{f}(\pi)$ will denote the bounded linear operator on the Hilbert space $H_{\pi}$, the representation space for $\pi$, given by

$$
\hat{f}(\pi)=\int_{G} f(x) \pi(x) d \mu(x),
$$

where the integral has to be suitably interpreted. $\hat{f}(\pi)$, which is also denoted $f(\pi)$ or $\pi(f)$, is the so-called operator valued Fourier transform of $f$. If $h \in L^{1} \cap L^{2}$ then $\{\pi \in \hat{G}: \hat{h}(\pi)=0\}$ is a measurable subset of $\hat{G}$ and so it makes sense to talk about $m(\{\pi: \hat{h}(\pi)=0\})$. If $g \in G$ and $h$ is a function on $G,{ }^{g} h$ and $h^{g}$ will denote functions on $G$ defined by ${ }^{g} h(x)=h\left(g^{-1} x\right)$ and $h^{g}(x)=h(x g)$. A set $E$, as above, is said to be a " $P$-set for $L^{1}(G)$ " if and only if: $f \in L^{1}(G)$ and $\int_{g_{1} E g_{2}} f d \mu=0 \forall g_{1}, g_{2} \in G$ implies that $f=0$ a.e. Finally for $h_{1}, h_{2} \in L^{1}(G)$, $h_{1} * h_{2}$ will denote the convolution

$$
\begin{aligned}
\left(h_{1} * h_{2}\right)(x) & =\int_{G} h_{1}(x g) h_{2}\left(g^{-1}\right) d \mu(g) \\
& =\int_{G} h_{1}\left(x g^{-1}\right) h_{2}(g) d \mu(g) .
\end{aligned}
$$

One knows that $\left(h_{1} * h_{2}\right) \curlyvee(\pi)=\hat{h}_{1}(\pi) \hat{h}_{2}(\pi)$ and $\left({ }^{g} h\right) \curlyvee(\pi)=\pi(g) \hat{h}(\pi)$.

\section{Some basic results.}

LEMMA 3.1. Let $E$ be a Borel set in $G$ with $0<\mu(E)<\infty$ and $f \in L^{1}(G)$. Then $\int_{g_{1} E g_{2}} f d \mu=0 \forall g_{1}, g_{2} \in G$ if and only if $\hat{1}_{\tilde{E}}(\pi) \pi(g) \hat{f}(\pi)=0 \forall \pi \in \hat{G}$ and $\forall g \in G$.

PROOF. It can be easily proved that the condition $\int_{g_{1} E g_{2}} f d \mu=0 \forall g_{1}, g_{2} \in G$ is equivalent to the condition $1_{\tilde{E}}{ }^{g} f \equiv 0 \forall g \in G$. By the injectivity of the Fourier transform, this in turn is equivalent to $\left(1_{\tilde{E}}{ }^{g} f\right)^{\wedge}(\pi)=0 \forall \pi \in \hat{G}, \forall g \in G$. The lemma then follows from the standard properties of the operator valued Fourier transform mentioned in $\S 2$.

Proposition 3.2. Let $E$ be as in Lemma 3.1. If $m\left(\left\{\pi \in \hat{G}: \hat{\mathbf{1}}_{\tilde{E}}(\pi)=0\right\}\right)=0$ then $E$ is a $P$-set for $L^{1}(G)$.

ProOF. Suppose $E$ satisfies the above condition and $f \in L^{1}(G)$ is such that $\int_{g_{1} E g_{2}} f d \mu=0, \forall g_{1}, g_{2} \in G$. Then we have to show that $f=0$ a.e. By Lemma 
$3.1 \hat{1}_{\tilde{E}}(\pi) \pi(g) \hat{f}(\pi)=0 \forall g \in G, \forall \pi \in \hat{G}$. Now for a fixed $\pi \in \hat{G}$, let $H_{\pi}$ be the Hilbert space on which $\pi$ acts. Since $\pi$ is irreducible, for any nonzero $v \in H_{\pi}$, $\operatorname{Span}\{\pi(g) v: g \in G\}$ is dense in $H_{\pi}$. Therefore if $\hat{1}_{\tilde{E}}(\pi) \neq 0$ we must have $\hat{f}(\pi)=0$. (Otherwise choose $v \in H_{\pi}$ such that $w=\hat{f}(\pi) v \neq 0$. Then since $\hat{1}_{\tilde{E}}(\pi) \neq 0$ and $\operatorname{span}\{\pi(g) w\}$ is dense in $H_{\pi}$, we must have $\hat{1}_{\tilde{E}}(\pi) \pi(g) w \neq 0$ for some $g \in G$ thus giving a contradiction.) Thus $\hat{f}(\pi)=0$ a.e. $\pi$ and therefore $f=0$ a.e. (For $h \in L^{1} \cap L^{2}$, the fact that $\hat{h}(\pi)=0$ a.e. $\pi$ implies $h=0$ a.e. follows from the abstract Plancherel theorem. That it continues to be valid for $h \in L^{1}$ follows from a simple argument involving convolution against an approximate identity.)

\section{Some applications of the basic results.}

(a) SEMISIMPLE LIE GROUPS. Let $G$ be a noncompact, connected, semisimple, rank-1 Lie group with finite centre. (For simplicity we assume $\operatorname{rank} G=1$, though this is not really necessary.) The example of a set $E$ which is not a $P$-set given in [Si II] depends on the existence of the discrete series. In this section we show how the existence of the discrete series is not really necessary to construct such examples, thus answering a question raised at the end of [Si II].

Let $G=K A N$ be an Iwasawa decomposition of $G$. Let $M$ be the centralizer of $A$ in $K . A$ is isomorphic to R. For $\sigma \in \hat{M}$ let $\left\{\pi_{\sigma, \lambda}\right\}_{\lambda \in \hat{A}}$ be the principal series of representations of $G$ (see [Wa]). If $G$ has discrete series let us denote the corresponding subset of $\hat{G}$ by $\hat{G}_{d}$. Then one knows that the Plancherel measure, $m$, for $G$ is "supported" on the principal series and $\hat{G}_{d}$ (moreover if $\pi \in \hat{G}_{d}, m(\pi)>0$ ). Now for certain groups one can pick $\delta \in \hat{K}$ for which the trivial representation of $M$ does not occur in $\left.\delta\right|_{M} .(\mathrm{SL}(2, \mathbf{R})$ and $\mathrm{SL}(2, \mathbf{C})$ are examples of such groups.) Let $\chi_{\delta}(k)=(\operatorname{dim} \delta)(\operatorname{trace} \delta(k))$ for $k \in K$ and $\delta \in \hat{K}$. Pick a nontrivial function $f \in L^{1}(G)$ with the property that $\bar{\chi}_{\delta} * f=f$. (That such functions exist can be shown in the case of $\operatorname{SL}(2, \mathbf{R})$ or $\operatorname{SL}(2, \mathbf{C})$.) For such $f$, one can show that $\hat{f}\left(\pi_{1, \lambda}\right)=0$ where 1 denotes the trivial representation of $M$. Now take $E$ to be a $K$-bi-invariant set in $G$ of positive finite measure. (It is actually enough to take $E$ invariant on one side by $K$.) Again for such a set one can show $\pi_{\sigma, \lambda}\left(1_{\tilde{E}}\right)=0$ if $\sigma$ is not the trivial representation of $M$. Also $\hat{1}_{\tilde{E}}(\pi)=0$ if $\pi \in \hat{G}_{d}$. Combining all this we have $\hat{1}_{\tilde{E}}(\pi) \pi(g) \hat{f}(\pi)=0 \forall g \in G$ and a.e. $\pi \in \hat{G}$. Thus $\int_{g_{1} E g_{2}} f(x) d \mu(x)=0$ $\forall g_{1}, g_{2} \in G$ follows from Lemma 3.1, i.e. $E$ is not a $P$-set for $L^{1}(G)$ since $f$ was chosen to be nontrivial. (Note: Lemma 3.1 continues to be valid if we replace " $\forall \pi \in \hat{G}, \forall g \in G$ " by "a.e. $\pi \in \hat{G}, \forall g \in G$ ".)

In particular, if $E$ is relatively compact the above shows that things are in sharp contrast to the case of $\mathbf{R}^{n}$ or a symmetric space of the noncompact type where any relatively compact set $E$ of positive measure is a $P$-set for $L^{1}$ (see [R I, Sa, Si II]).

In view of the "holomorphy" of the Fourier transform for $L^{1}$ functions on a semisimple Lie group one can restate Proposition 3.2 for noncompact semi simple $G$ as follows: If for each $\sigma \in \hat{M} \exists \lambda_{\sigma} \in \hat{A}$ with $\pi_{\sigma, \lambda_{\sigma}}\left(1_{\tilde{E}}\right) \neq 0$ and $\pi\left(1_{\tilde{E}}\right) \neq 0 \forall \pi \in \hat{G}_{d}$ then $E$ is a $P$-set for $L^{1}(G)$. However what would be interesting is to apply this to obtain a geometric criterion for $E$ to be a $P$-set for $L^{1}(G)$. For example if $\Gamma$ is a discrete subgroup of $G$ with volume $(G / \Gamma)<\infty$, then is a fundamental domain for $G / \Gamma$ a $P$-set for $L^{1}$ ? Notice that this is analogous to the case of a rectangle in $\mathbf{R}^{2}$. 
(b) COMPACT GROUPS. In this case $\hat{G}$ is discrete (though not necessarily countable) and for $\pi \in \hat{G}, m(\pi)=\operatorname{dim}(\pi)>0$. Using the Peter-Weyl theorem we can strengthen Proposition 3.2 to read: $E$ is a $P$-set for $L^{1}$ iff $\hat{1}_{\tilde{E}}(\pi) \neq 0 \forall \pi \in \hat{G}$.

Suppose $G$ is a compact Lie group. Equip $G$ with a bi-invariant Riemannian structure and fix a positive number $R_{0}$ such that for any $s<R_{0}$, the exponential mapping is an analytic diffeomorphism from the open ball of radius $s$ around 0 onto the open geodesic ball of radius $s$ around $e$. Using a real analyticity argument along the lines used in [Be-Z] for compact symmetric spaces of rank-1, one can show that for fixed $\pi \in \hat{G}$, the mapping $r \rightarrow \hat{1}_{B_{r}}(\pi)$ is a nontrivial real analytic function of $r$ in the interval $\left[0, R_{0}\right)$ and hence can vanish on only a countable set $S_{\pi}$ of values for $r$. (Here $B_{r}$ is the geodesic ball in $G$ of radius $r$ about $e$. Also since $\pi(e)=I$, $\hat{1}_{B_{r}}(\pi)$ is a nontrivial function of $r$.) Since $G$ is a Lie group $\hat{G}$ is countable and so it will follow that for $r$ in $\left[0, R_{0}\right) \backslash S, \hat{1}_{B_{r}}(\pi) \neq 0 \forall \pi \in \hat{G}$, where $S=\bigcup S_{\pi}$. Thus for all such $r, B_{r}$ is a $P$-set for $L^{1}$. (In the case of compact symmetric spaces of rank-1, $[\mathbf{B e}-\mathbf{Z}]$ were actually able to identify explicitly the exceptional countable set $S$.)

Now suppose $G$ is a compact group for which $\hat{G}$ is not countable. Then for any $E$ of positive measure in $G$, by the Peter-Weyl theorem,

$$
\left\|1_{\tilde{E}}\right\|_{2}^{2}=\sum_{\delta \in \hat{G}} \operatorname{tr}\left(\hat{1}_{\tilde{E}}(\delta) * \hat{1}_{\tilde{E}}(\delta)\right) d(\delta)<\infty .
$$

Since $\hat{G}$ is not countable, we have $\operatorname{tr}\left(\hat{1}_{\tilde{E}}(\delta) * \hat{1}_{\tilde{E}}(\delta)\right)=0$ for all but countably many $\delta$. Equivalently $\hat{1}_{\tilde{E}}(\delta)=0$ for all but countably many $\delta$. So $E$ cannot be a $P$-set. Thus, in this case, there are no $P$-sets.

In [R II] Rana asks the question whether in an arbitrary locally compact, second countable group, determining sets exist for the left action. In fact we do not know the answer to this question even if we consider, as in this paper, two-sided action. However, the example above shows that things can be quite bad if we do not assume second countability.

(c) The Heisenberg group $H_{n}$. The Heisenberg group $H_{n}$ is a simply connected nilpotent Lie group and consists of triples $(p, q, t)$ with $p, q \in \mathbf{R}^{n}$ and $t \in \mathbf{R}$ (as a set $H_{n}$ is just $\mathbf{R}^{2 n+1}$ ). Multiplication is defined by

$$
(p, q, t) \cdot\left(p^{\prime}, q^{\prime}, t^{\prime}\right)=\left(p+p^{\prime}, q+q^{\prime}, t+t^{\prime}+\left(p q^{\prime}-p^{\prime} q\right) / 2\right)
$$

where $p q$ denotes the usual dot product in $\mathbf{R}^{n}$. The Haar measure $d \mu$ is just $d p d q d t$. For each $h \in \mathbf{R} \backslash\{0\}$, one can define an irreducible unitary representation $\pi_{h}$ on $L^{2}\left(\mathbf{R}^{n}\right)$ by: $\left(\left(\pi_{h}(p, q, t)\right) f\right)(x)=e^{2 \pi i q x+\pi i h p q+2 \pi i h t} f(x+h p) . \quad\left\{\pi_{h}\right\}_{h \in \mathbf{R} \backslash\{0\}}$ is a family of inequivalent irreducible unitary representations and in fact the Plancherel measure is supported on this family and is given by $d m=|h|^{n} d h$. If $g \in C_{c}^{\infty}\left(H_{n}\right)$, $\pi_{h}(g)$ is given by an integral operator with kernel $K_{h}(x, w)$ given by

$$
K_{h}(x, w)=\frac{1}{|h|^{n}}\left(\mathscr{F}_{23} g\right)\left(\frac{w-x}{h}, \frac{w+x}{2}, h\right)
$$

where $\mathscr{F}_{23} g$ is the ordinary (Euclidean) Fourier transform of $g$ in the second and third variables. Exploiting the above connection with the ordinary Fourier transform and using analyticity properties of the ordinary Fourier transform for compactly supported functions, one can easily prove: If $g \in C_{c}^{\infty}\left(H_{n}\right)$ and $g \neq 0$ then $m\left(\left\{h: \pi_{h}(g)=0\right\}\right)=0$. A slight modification of this leads to the following: 
LEMMA 4.1. If $g$ is a nontrivial bounded measurable compactly supported function on $H_{n}$ then $m\left(\left\{h: \pi_{h}(g)=0\right\}\right)=0$.

In view of Proposition 3.2 the following corollary is immediate.

COROLlaRY 4.2. Let $E$ be a bounded (i.e. $\bar{E}$ compact) Borel set of positive Haar measure in $H_{n}$. Then $E$ is a $P$-set for $L^{1}\left(H_{n}\right)$.

We conclude this section with the following question: Does the above hold for any simply connected nilpotent Lie group?

For details about the representations of nilpotent Lie groups and $H_{n}$, see [L] and [T].

(d) The Euclidean motion group on the Plane. For $p \in \mathbf{R}^{2}$ and $A \in \mathrm{SO}(2)$ let $\tau_{p, A}$ be the rigid motion of $\mathbf{R}^{2}$ defined by $\tau_{p, A}(v)=A v+p, v \in \mathbf{R}^{2}$. Let $G=\left\{\tau_{p, A}: p \in \mathbf{R}^{2}, A \in \mathrm{SO}(2)\right\}$. The group multiplication is composition of rigid motions and $G$ as a manifold is diffeomorphic to $\mathbf{R}^{2} \times \mathrm{SO}(2)$. The Haar measure is $d p d k$ where $d p$ is Lebesgue measure on $\mathbf{R}^{2}$ and $d k$ is Haar measure on $\mathrm{SO}(2)$. Let $\left\{\pi_{h}\right\}_{h \in \mathbf{R}^{+}}$be the "principal series" representations of $G$ (see $[\mathbf{S u}]$ for details). The "principal series", each of which is realized on $L^{2}(\mathrm{SO}(2))$, is a subset of $\hat{G}$ that supports the Plancherel measure $m$. If one examines the proof of the Paley-Wiener theorem for $G$ proved in [Su] one finds: if $g \in C_{c}^{\infty}(G)$ and $g \neq 0$, then $m\left(\left\{h: \pi_{h}(g)=0\right\}\right)=0$. A slight modification of this once again yields:

LEMMA 4.3. If $g$ is a nontrivial bounded measurable compactly supported function on $G$, then $m\left(\left\{h: \pi_{h}(g)=0\right\}\right)=0$.

Consequently we have

COROLlaRY 4.4. If $E$ is a bounded (i.e. $\bar{E}$ compact) measurable subset of $G$ of positive Haar measure, then $E$ is a $P$-set for $L^{1}(G)$.

Again we end this section with a question of what happens to motion groups in general? (By a motion group we mean a semidirect product of a compact group and a vector group.)

5. Concluding remarks. We hope that this paper demonstrates that much needs to be done regarding the Pompeiu problem for groups. For example what happens if we drop the integrability condition on the functions? From [Be II] one understands that these questions are related to questions about mean periodic functions on unimodular groups - again an area where much needs to be done. See also [We]. Also it is clear from the above examples that the Pompeiu problem on a given group $G$ is very much related to the "structure and nature" of $\hat{G}$.

ACKNOWLEDGMENTS. We would like to thank G. Folland and S. C. Bagchi for teaching us the representation theory of nilpotent Lie groups and in particular the Heisenberg groups. D. Scott wishes to thank the University of Puget Sound for the John Lantz Fellowship which supported his work and wishes to thank I. S. I. Bangalore for their hospitality.

\section{REFERENCES}

[Ba-Si] S. C. Bagchi and A. Sitaram, Determining sets for measures on $\mathbf{R}^{n}$, Illinois J. Math. 26 (1982), 419-422.

[Be I] C. A. Berenstein, An inverse spectral theorem and its relation to the Pompeiu problem, J. Analyse Math. 37 (1980), 128-144. 
[Be II] C. A. Berenstein, Spectral synthesis on symmetric spaces, Contemp. Math. Amer., vol. 63, Math. Soc., Providence, R.I., 1987, pp. 1-25.

[Be-Sh] C. A. Berenstein and M. Shahshahani, Harmonic analysis and the Pompeiu problem, Amer. J. Math. 105 (1983), 1217-1229.

[Be-Z] C. A. Berenstein and L. Zalcman, The Pompeiu problem in symmetric spaces, Comment. Math. Helv. 55 (1980), 593-621.

[Br-S-T] L. Brown, B. M. Schreiber and B. A. Taylor, Spectral synthesis and the Pompeiu problem, Ann. Inst. Fourier 23 (1973), 125-154.

[L] R. Lipsman, Group representations, Lecture Notes in Math., vol. 388, Springer, 1974.

[R I] I. K. Rana, Determination of probability measures through group actions, $\mathrm{Ph} . \mathrm{D}$. thesis, Indian Statistical Institute, 1979.

[R II] I. K. Rana, Determination of probability measures through group actions, Zeit Wahr,53 (1980), 197-206.

[Sa] N. A. Sapagov, A uniqueness problem for finite measures in Euclidean spaces-problems in the theory of probability distributions, Zap. Nauchn. Sem. (LOMI) Leningrad, vol. 41, 1974, pp. 3-13.

[Si I] A. Sitaram, Fourier analysis and determining sets for Radon measures on $\mathbf{R}^{n}$, Illinois J. Math. 28 (1984), 339-347.

[Si II] A. Sitaram, Some remarks on measures on noncompact semisimple Lie groups, Pacific J. Math. 110 (1984), 429-434.

[Su] M. Sugiura, Unitary representations and harmonic analysis-an introduction, Kodansha, 1975.

[T] M. E. Taylor, Noncommutative harmonic analysis, Math. Surveys and Monos., vol. 22, Amer. Math. Soc., Providence, R.I., 1986.

[Wa] G. Warner, Harmonic analysis on semisimple Lie groups, 2 vols., Springer-Verlag, 1972.

[We] Y. Weit, On Schwartz's theorem for the motion group, Ann. Inst. Fourier 30 (1980), 91-107.

[Z] L. Zalcman, Offbeat integral geometry, Amer. Math. Monthly 87 (1980), 161-175.

Department of Mathematics, University of Puget Sound, Tacoma, WashINGTON 98416

Stat-Math Unit, indian Statistical Institute, R.V. College Post Office, BANGALORE 560059, INDIA 